\title{
Mechanisms for Coronal Mass Supply by Evaporative Micro-Events
}

\author{
J. C. Brown \\ Department of Physics \& Astronomy, University of Glasgow, G128QQ, \\ Scotland, UKUniversity of Glasgow
}

S. Krucker

Space Sciences Laboratory, University of California, Berkeley, CA 94720-7450, USA

M. Güdel

Paul Scherrer Institute, CH-5232, Villigen PSI, Switzerland

\author{
A. O. Benz \\ Swiss Federal Institute of Technology, Institute of Astronomy, \\ ETH-Zentrum, CH-8092 Zürich, Switzerland
}

\begin{abstract}
There is extensive evidence from SoHO and other data that "micro-events" play an important role in sustaining at least some components of the solar corona. These are often termed coronal micro-"heating events" though a major part of their role is feeding coronal loops through chromospheric evaporation. We consider what can be learnt from these data concerning the energy release and transport mechanisms driving the evaporation, including thermal conduction and fast particles. We conclude, from one large event and the statistics of many small ones, that conductive evaporation alone does not fit observations and that fast particles or some other nonthermal driver must be involved.
\end{abstract}

\section{Introduction}

Krucker and Benz (2000) (KB) discussed SOHO EIT microevents and estimated the increase in coronal loop $E M, T$ values during one of the largest in order to study heating and mass supply mechanisms. From the whole line-of-sight projected area, values in the loop itself have to be derived using assumptions on what fraction $\phi$ the pre-event loop $E M$ contributes to the observed total flux. We find that the effect of varying $\phi$ over $0 \rightarrow 1$ is about $7 \%$ on the derived $T$ (enough to affect conductive flux substantially), while for the mass and thermal energy added to the loop the uncertainties are factors of order 2 , both mass and energy decreasing for increasing $\phi$. We therefore examined the requirements of evaporation mechanism for the extreme cases $\phi=0,1$. 


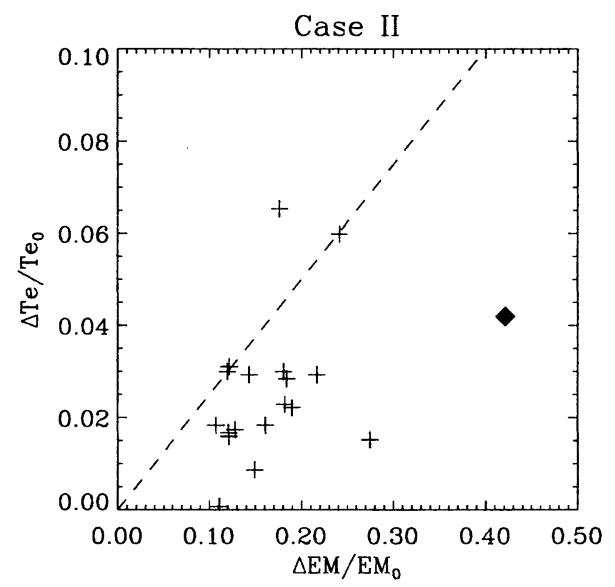

Figure 1. Relationship between fractional loop $T_{o}$ and $E M$ enhancements predicted by the RVT conductive scaling law, with observed microevents superposed.

\section{Conductive evaporation}

Hydrostatic loops dominated by thermal conduction and radiation were discussed extensively by Rosner et al. (1978) (RVT) and others. Their scaling law links the high loop-top temperature $T_{o}$ (generated by coronal heating), length $L$ and loop density $n_{o}$. From this it follows that conductive evaporation loop changes should satisfy

$$
E M_{2} / E M_{1}=\left(T_{o 2} / T_{o 1}\right)^{4}
$$

In Fig. 1 we plot fractional increases in $T_{o}, E M$ against one another for the events observed by KB using EIT (the largest event indicated by the diamond). The dashed line is the expected relationship for conduction dominated evaporation, in the case where no background fluxes have been subtracted to get the loop emission $(\phi=1)$. Results for $\phi=0$ are not very different. It is clear that observed microevent $E M$ enhancements are generally much larger than predicted by the hydrostatic conductive loop theory.

\section{Nonthermal evaporation}

To examine how much nonthermal energy flux $F_{c}$ is needed to evaporate the large $E M$ increases found above we treated evaporation similarly to Brown's (1973) flare modeling but with: finite and changing loop top pressure - this increases $F_{c}$ since it raises chromospheric density; a parametric heating function intended to describe a wider range of mechanisms than particle beams; ambient chromospheric heating term (small in large flares - this substantially reduces the $F_{c}$ requirement). Fig. 2 shows the $F_{c}$ required to produce a nonthermal evaporative enhancement in coronal column density by a factor $N_{c 2} / N_{c 1}$ for an appropriate pre-event chromosphere and for the case $\phi=1$. Curves are labeled with the index $\beta$ of variation of heating rate $N^{-\beta}$ with column depth $N$ into the 


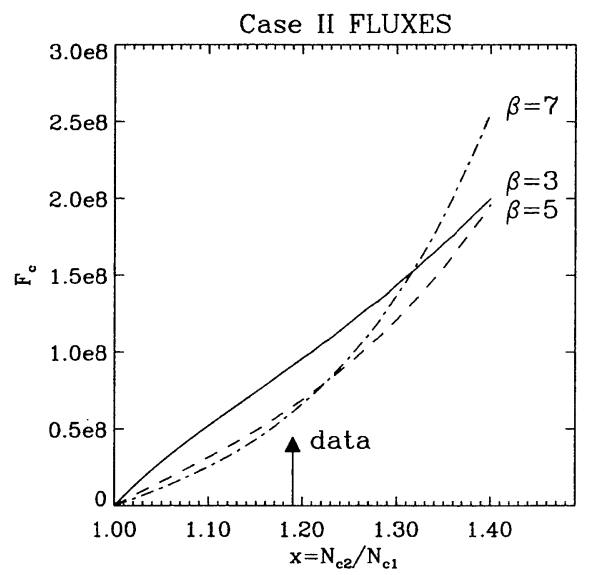

Figure 2. Nonthermal flux $F_{c}$ needed to yield a prescribed loop column density enhancement for $\phi=1$.

chromosphere. The $N_{c 2} / N_{c 1}$ factor inferred from the observed large microevent of Fig. 1 is indicated.

\section{Conclusions}

We conclude (cf. Brown et al. 2000) that the coronal loop mass supply in SOHO EIT microevents cannot result from conductive evaporation from coronal loop heating alone but must involve nonthermal heating of the upper chromosphere either in situ or by injection of nonthermal energy (e.g. particles) from the coronal loop. Combined data from HESSI and SOHO should provide tests of the particle hypothesis.

Acknowledgments. This work was supported by UK PPARC Research and Travel Grants and by Visitor funds from ETH Institut für Astronomie, Zürich.

\section{References}

Brown, J.C. 1973, Solar Phys., 31, 143

Brown, J.C., Krucker, S., Güdel, M., \& Benz, A.O. 2000, A\&A, 359, 1185

Krucker, S. \& Benz, A.O. 2000, Solar Phys., 191, 343

Rosner, R., Tucker, W.H., \& Vaiana, G.S. 1978, ApJ, 220, 643 